\title{
The coordinate-wise core for multiple-type housing markets is second-best incentive compatible
}

Citation for published version (APA):

Klaus, B. E. (2005). The coordinate-wise core for multiple-type housing markets is second-best incentive compatible. METEOR, Maastricht University School of Business and Economics. METEOR Research Memorandum No. 019 https://doi.org/10.26481/umamet.2005019

Document status and date:

Published: 01/01/2005

DOI:

10.26481/umamet.2005019

Document Version:

Publisher's PDF, also known as Version of record

\section{Please check the document version of this publication:}

- A submitted manuscript is the version of the article upon submission and before peer-review. There can be important differences between the submitted version and the official published version of record.

People interested in the research are advised to contact the author for the final version of the publication, or visit the DOI to the publisher's website.

- The final author version and the galley proof are versions of the publication after peer review.

- The final published version features the final layout of the paper including the volume, issue and page numbers.

Link to publication

\footnotetext{
General rights rights.

- You may freely distribute the URL identifying the publication in the public portal. please follow below link for the End User Agreement:

www.umlib.nl/taverne-license

Take down policy

If you believe that this document breaches copyright please contact us at:

repository@maastrichtuniversity.nl

providing details and we will investigate your claim.
}

Copyright and moral rights for the publications made accessible in the public portal are retained by the authors and/or other copyright owners and it is a condition of accessing publications that users recognise and abide by the legal requirements associated with these

- Users may download and print one copy of any publication from the public portal for the purpose of private study or research.

- You may not further distribute the material or use it for any profit-making activity or commercial gain

If the publication is distributed under the terms of Article $25 \mathrm{fa}$ of the Dutch Copyright Act, indicated by the "Taverne" license above, 


\title{
The Coordinate-Wise Core for Multiple-Type Housing Markets is Second-Best Incentive Compatible
}

\author{
Bettina Klaus*
}

May 2006

\begin{abstract}
We consider the generalization of Shapley and Scarf's (1974) model of trading indivisible objects (houses) to so-called multiple-type housing markets. We show that the prominent solution for these markets, the coordinate-wise core rule, is second-best incentive compatible. In other words, there exists no other strategy-proof trading rule that Pareto dominates the coordinate-wise core rule. Given that for multipletype housing markets Pareto efficiency, strategy-proofness, and individual rationality are not compatible, we show that applying the coordinate-wise core rule is a minimal concession with respect to Pareto efficiency while preserving strategy-proofness and individual rationality.
\end{abstract}

Keywords: coordinate-wise core, indivisible goods, second-best incentive compatibility, strategy-proofness.

JEL classification: D63, D70

\section{Introduction}

We consider the generalization of Shapley and Scarf's (1974) model of trading indivisible objects (houses) to so-called multiple-type housing markets. In Shapley and Scarf's (1974) housing markets each agent is endowed with an indivisible commodity, for instance a house. Furthermore, each agent wishes to consume exactly one house and has a strict ranking of all houses in the market. Important real-life applications of Shapley and Scarf's (1974) model are indeed concerned with the allocation or reallocation of housing, for instance student housing (e.g., Abdulkadiroğlu and Sönmez, 1999, Chen and Sönmez, 2002,2004, and Sönmez and Ünver, 2005). Interestingly, one of the best known solution concepts for barter economies can always be applied: the core for any housing market is non-empty (Scarf and Shapley, 1974). In addition, the core is always a singleton and it coincides with the unique competitive allocation (Roth and Postlewaite, 1977). Furthermore, the trading rule that assigns the unique core allocation for any housing market is strategy-proof, i.e., no agent can benefit from misrepresenting his preferences (Roth, 1982). In addition, Ma (1994) demonstrated that the core rule is the unique trading rule satisfying Pareto efficiency, strategy-proofness, and individual rationality, i.e., no agent is worse off after

\footnotetext{
*Department of Economics, Maastricht University, P.O. Box 616, 6200 MD Maastricht, The Netherlands, e-mail: B.Klaus@algec.unimaas.nl. I thank Fuhito Kojima and William Thomson for their comments.
} 
trading. For a more general class of problems, Sönmez's (1999) results imply Ma's (1994) characterization of the core rule. Recently, Miyagawa (2002a) complemented Ma's (1994) result by essentially characterizing the core rule by anonymity (the names of the agents do not matter), strategy-proofness, individual rationality, and non-bossiness (no agent can influence another agent's final consumption without changing his final consumption). ${ }^{1}$

We consider an extension of Shapley and Scarf's (1974) housing markets in which there are several types of indivisible commodities, maybe houses and cars, each agent is endowed with an indivisible commodity of each type and wishes to consume exactly one commodity of each type. A more realistic example would be the situations of students' enrollment at many universities where courses are taught in parallel sections. A good example for such a situation is Maastricht University where small classes are part of the university's teaching philosophy of problem oriented learning. Typically, courses are largely based on the students' work in so-called tutor groups that are strictly limited to an enrollment of at most 14 students. So, after the registration process, each student is enrolled in exactly one tutor group for each course. Tutor groups are typically scheduled at different times of the day, so students may have an interest to trade tutor groups among each others to adjust their schedule to their preferences. At Maastricht University, course coordinators typically accommodate changes in the tutor groups as long as the upper limit of 14 students per tutor group is not affected. Clearly, only tutor groups of the same course can be traded.

We stick to the classical nomenclature and call these markets multiple-type housing markets. Moulin (1995) introduced multiple-type housing markets, but Konishi et al. (2001) were the first to analyze the model. They demonstrate that when increasing the dimension of the model by adding other types of indivisible commodities, most of the positive results obtained for the one-dimensional case disappear: even for additively separable $^{2}$ preferences the core may be empty and no Pareto efficient, strategy-proof, and individually rational trading rule exists. For separable preferences, Konishi et al. (2001) and Wako (2005) suggested an alternative solution to the core by first using separability to decompose a multiple-type housing market into "coordinate-wise submarkets" and second, determining the core in each submarket. Wako (2005) calls the resulting outcome the commodity-wise competitive allocation and shows that it is implementable in coalition-proof Nash equilibria, but not in strong Nash equilibria. We call the rule that assigns the commodity-wise competitive allocation, or equivalently the core allocation, in each submarket the "coordinate-wise core rule." From its definition it follows easily that the coordinate-wise core rule satisfies strategy-proofness and individual rationality, but not Pareto efficiency. In a recent paper Miyagawa (2002b) characterizes the coordinatewise core rule by citizen sovereignty, ${ }^{3}$ strategy-proofness, individual rationality, and nonbossiness. Hence, in the absence of Pareto efficient, strategy-proof, and individual rational trading rules, the coordinate-wise core rule seems to be a good compromise.

In this article, we would like to further promote the coordinate-wise core rule as a desirable solution for multiple-type housing markets. We do so by showing that the coordinate-wise core is second-best incentive compatible (Theorem 1). In other words, there exists no other strategy-proof trading rule that Pareto dominates the coordinate-

\footnotetext{
${ }^{1}$ The only other rule satisfying these properties is the "no-trade rule."

${ }^{2}$ By separability, preferences between commodities of the same type do not depend on the consumption of commodities of different types. We formally introduce separable preferences in Section 2.

${ }^{3}$ No allocation is excluded from the range of the trading rule.
} 
wise core rule. Given that for multiple-type housing markets Pareto efficiency, strategyproofness, and individual rationality are not compatible, by Theorem 1 we show that applying the coordinate-wise core rule is a minimal concession with respect to Pareto efficiency while preserving strategy-proofness and individual rationality.

\section{Multiple-Type Housing Markets and the Coordinate-wise Core}

We mostly follow Miyagawa's (2002b) model and notation of housing markets with multiple types. Let $N=\{1, \ldots, n\}, n \geq 2$, be the set of agents. There exist $\bar{\ell} \geq 1$ types of (distinct) indivisible objects. The set of object types is denoted by $L=\{1, \ldots, \bar{\ell}\}$ and each agent $i \in N$ is endowed with one object of each type $\ell \in L$, denoted by $i$. Thus, $N$ also denotes the set of objects of each type.

An allocation is a reallocation of objects among agents such that each agent again receives one object of each type. Formally, an allocation is a list $x=\left(x_{i}(\ell)\right)_{i \in N, \ell \in L} \in$ $N^{N \times L}$ such that

(i) each agent receives one object of each type, i.e., for all $i \in N$ and all $\ell \in L, x_{i}(\ell) \in N$ denotes the object of type $\ell$ that agent $i$ consumes, e.g., if $x_{i}(\ell)=j$, then agent $i$ receives agent $j$ 's endowment of type $\ell$, and

(ii) no object of any type is assigned to more than one agent at allocation $x$. Thus, for all $\ell \in L, \cup_{i \in N}\left\{x_{i}(\ell)\right\}=N$.

Let $X$ denote the set of allocations. Given $x \in X$ and $\ell \in L, x(\ell)=\left(x_{1}(\ell), \ldots, x_{n}(\ell)\right)$ denotes the allocation of type- $\ell$ objects. Given $x \in X$ and $i \in N, x_{i}=\left(x_{i}(1), \ldots, x_{i}(\bar{\ell})\right)$ denotes the list of objects that agent $i$ receives at allocation $x$. We call $x_{i}$ agent $i$ 's (consumption) bundle. Note that the set of bundles for each agent $i \in N$ can be denoted by $N^{L}$. We denote each agent $i$ 's endowment by $(i, \ldots, i) \in N^{L}$.

Each agent $i \in N$ has complete, transitive, and strict preferences $R_{i}$ over bundles, i.e., $R_{i}$ is a linear order over $N^{L}$. Thus, for bundles $x_{i}, y_{i} \in N^{L}, x_{i} P_{i} y_{i}$ implies $x_{i} \neq y_{i}$ and $x_{i} I_{i} y_{i}$ implies $x_{i}=y_{i}$. In addition to being linear orders, we assume that preferences are separable: each agent $i \in N$ has complete, transitive, and strict marginal preferences $R_{i}(\ell)$ over the objects of each type $\ell$ and prefers consuming a bundle $x_{i}$ to a bundle $y_{i}$ if $x_{i} \neq y_{i}$ and all objects received at $x_{i}$ are (weakly) better than those received at $y_{i}$ according to the marginal preferences, i.e., for all $\ell \in L, x_{i}(\ell) R_{i}(\ell) y_{i}(\ell)$. Formally, a preference relation $R_{i}$ over $N^{L}$ is separable if for all $\ell \in L$, there exists a linear order $R_{i}(\ell)$ defined over $N, P_{i}(\ell)$ being its strict part, such that for any two bundles $x_{i}, y_{i} \in N^{L}$, if for all $\ell \in L x_{i}(\ell) R_{i}(\ell) y_{i}(\ell)$, and for some $\tilde{\ell}, x_{i}(\tilde{\ell}) P_{i}(\tilde{\ell}) y_{i}(\tilde{\ell})$, then $x_{i} P_{i} y_{i}$. By $\mathcal{R}$ we denote the set of separable linear orders over $N^{L}$. Since for all agents $i \in N, \mathcal{R}$ represents agent $i$ 's set of preferences, by $\mathcal{R}^{N}=\times_{i \in N} \mathcal{R}$ we denote the set of (preference) profiles. Since the set of agents and their endowments remain fixed throughout, $\mathcal{R}^{N}$ also denotes the set of housing markets with multiple types, or the set of multiple-type housing markets. For $\bar{\ell}=1$ our model of multiple-type housing markets equals the classical Shapley and Scarf (1974) housing market model.

A (trading) rule (for multiple-type housing market) is a function $\varphi: \mathcal{R}^{N} \rightarrow X$ that assigns to each multiple-type housing market $R \in \mathcal{R}^{N}$ an allocation $\varphi(R) \in X$. By $\varphi_{i}(R)$ we denote the bundle assigned by $\varphi$ to agent $i \in N$. 
Before we introduce our main rule, the coordinate-wise core rule, we need some notation. The set of all reallocations of objects among the members of coalition $S \subseteq N$ is denoted by

$$
X_{S}=\left\{\left(x_{i}(\ell)\right)_{i \in S, \ell \in L} \in N^{S \times L}: \text { for all } \ell \in L, \cup_{i \in S}\left\{x_{i}(\ell)\right\}=S\right\} .
$$

Similarly, for $\ell \in L$ the set of all reallocations of type- $\ell$ objects among the members of coalition $S \subseteq N$ is denoted by

$$
X_{S}(\ell)=\left\{\left(x_{i}(\ell)\right)_{i \in S} \in N^{S}: \cup_{i \in S}\left\{x_{i}(\ell)\right\}=S\right\} .
$$

Given $x \in X$ and $\ell \in L$, a trading cycle for $x(\ell)$ is a coalition $T \subseteq N$ such that

(i) agents in $T$ obtain their objects of type $\ell$ by reallocating their endowments of type $\ell$ among themselves, i.e., $\left(x_{i}(\ell)\right)_{i \in T} \in X_{T}(\ell)$ and

(ii) coalition $T$ is minimal, i.e., there exists no $T^{\prime} \varsubsetneqq T$ such that $\left(x_{i}(\ell)\right)_{i \in T^{\prime}} \in X_{T^{\prime}}(\ell)$.

Note that for all $x \in X$ and $\ell \in L$, there exists a partition $\left\{T_{1}, \ldots, T_{m}\right\}$ of $N$ such that for each $k \in\{1, \ldots, m\}, T_{k}$ is a trading cycle for $x(\ell)$.

An allocation is in the (strict or strong) core if no coalition of agents can improve their welfare by reallocating their endowments among themselves. Formally, an allocation $x \in X$ is a core allocation for the multiple-type housing market $R \in \mathcal{R}^{N}$ if there exist no coalition $S \subseteq N$ and no $y \in X_{S}$ such that for all $i \in S, y_{i} R_{i} x_{i}$, and for some $j \in S$, $y_{j} P_{j} x_{j}$.

For a housing market with one object type, Shapley and Scarf (1974) showed that a core allocation always exists. Furthermore, Roth and Postlewaite (1977) proved that the set of core allocations for any housing markets with one object type equals a singleton. Using the so-called top-trading algorithm (due to David Gale, see Shapley and Scarf, 1974) one can easily calculate the unique core allocation for any housing market with one object type. For completeness, we give a description of the well-known top trading algorithm. Let $\bar{\ell}=1$ and $R \in \mathcal{R}^{N}$.

Step 1 Imagine that all agents are in a room together and each agent points at the agent who owns his most preferred object. If an agent $i \in N$ points to himself, then $\{i\}$ is a trading cycle in which agent $i$ keeps his endowment $i$. If a group of agents $S \subseteq N$ points at each other (e.g., $s_{1}$ points at $s_{2}, s_{2}$ points at $s_{3}$, and $s_{3}$ points at $s_{1}$ ), then they form a trading cycle $S$ in which they exchange objects accordingly, i.e., each agent in $S$ receives his most preferred object (e.g., agent $s_{1}$ receives object $s_{2}$, agent $s_{2}$ receives object $s_{3}$, and agent $s_{3}$ receives object $s_{1}$ ). Step 1 yields trading cycles $T_{1}, \ldots, T_{m(1)}, m(1) \geq 1$, and an assignment of objects to all trading agents in $\cup_{\tilde{m} \in\{1, \ldots, m(1)\}} T_{\tilde{m}}$. All agents in $\cup_{\tilde{m} \in\{1, \ldots, m(1)\}} T_{\tilde{m}}$ leave with their consumptions. If there are agents left, i.e., $N \backslash\left(\cup_{\tilde{m} \in\{1, \ldots, m(1)\}} T_{\tilde{m}}\right) \neq \emptyset$, move to the next step.

Step $k+1$ Each agent points at the agent who owns his most preferred object among the objects that are left. Similarly as before, Step $k+1$ yields trading cycles $T_{m(k)+1}, \ldots, T_{m(k+1)}, m(k+1) \geq m(k)+1$, and an assignment of objects to all trading agents in $\cup_{\tilde{m} \in\{m(k)+1, \ldots, m(k+1)\}} T_{\tilde{m}}$. All agents in $\cup_{\tilde{m} \in\{m(k)+1, \ldots, m(k+1)\}} T_{\tilde{m}}$ leave the room with their consumptions. If there are agents left, i.e., $N \backslash\left(\cup_{\tilde{m} \in\{1, \ldots, m(k+1)\}} T_{\tilde{m}}\right) \neq \emptyset$, move to the next step. 
After a finite number of steps $(\leq n)$, the top trading algorithm determines a partition of $N$ into trading cycles $T_{1}, \ldots, T_{\bar{k}}$ and the unique (core) allocation $x$ induced by these trading cycles.

For multiple-type housing markets with more than one object type, i.e., $\bar{\ell} \geq 2$, Konishi et al. (2001) demonstrated that the core may be empty. Furthermore, if the core is not empty, then it may not be a singleton. However, using the separability of preferences and the fact that a unique core allocation exists in each separate "marginal object type market," we now define the coordinate-wise core rule $\varphi^{c c}$. Konishi et al. (2001) mention the possibility to calculate the unique commodity-wise (strict) core for multiple-type housing markets with separable preferences. Wako (2005) introduced the coordinate-wise core under the name commodity-wise competitive allocations. Another article on the coordinate-wise core rule we briefly discuss later is Miyagawa (2002b).

The coordinate-wise core rule $\varphi^{c c}$ assigns to each multiple-type housing market $R \in \mathcal{R}^{N}$ the unique coordinate-wise core allocation $\varphi^{c c}(R) \equiv x \in X$ that is obtained by separately calculating the core allocation $x(\ell)$ for each object type $\ell \in L$ in its associated marginal object type market, e.g., by applying the top trading algorithm. Formally, for all $\ell \in L$, there exists no coalition $S \subseteq N$ and no $y(\ell) \in X_{S}(\ell)$, such that for all $i \in S, y_{i}(\ell) R_{i}(\ell) x_{i}(\ell)$, and for some $j \in S, y_{j}(\ell) P_{j}(\ell) x_{j}(\ell)$. For $\bar{\ell}=1$ we call $\varphi^{c c}$ the core rule.

We illustrate the coordinate-wise core rule in the following example.

Example 1. Let $N=\{1,2,3,4,5\}$ and $L=\{1,2\}$. Let $R \in \mathcal{R}^{N}$ such that marginal preferences are as listed in Table 1; i.e., for $i \in N$ and $l \in L$ each column labeled $R_{i}(l)$ represents agent $i$ 's marginal preferences for object type $l$ (objects that are more preferred are listed above less preferred objects).

\begin{tabular}{|c|c||c|c||c|c||c|c||c|c|}
\hline$R_{1}(1)$ & $R_{1}(2)$ & $R_{2}(1)$ & $R_{2}(2)$ & $R_{3}(1)$ & $R_{3}(2)$ & $R_{4}(1)$ & $R_{4}(2)$ & $R_{5}(1)$ & $R_{5}(2)$ \\
\hline \hline 2 & 5 & 3 & 5 & 1 & 5 & 5 & 5 & 2 & 5 \\
4 & 2 & 5 & 3 & 2 & 2 & 1 & 3 & 1 & 3 \\
5 & 4 & 4 & 1 & 3 & 1 & 3 & 1 & 4 & 2 \\
3 & 1 & 1 & 2 & 5 & 4 & 2 & 4 & 5 & 4 \\
1 & 3 & 3 & 4 & 4 & 3 & 4 & 2 & 3 & 1 \\
\hline
\end{tabular}

Table 1: Marginal Preferences for Example 1.

Step 1 yields for object type $1, \varphi^{c c}(R)(1)=(2,3,1, \cdot, \cdot)$, and for object type 2 , $\varphi^{c c}(R)(2)=(\cdot, \cdot, \cdot, \cdot, 5)$.

Step 2 yields for object type $1, \varphi^{c c}(R)(1)=(2,3,1,5,4)$, and for object type 2 , $\varphi^{c c}(R)(2)=(\cdot, 3,2, \cdot, 5)$.

Step 3 yields for object type $2, \varphi^{c c}(R)(2)=(4,3,2,1,5)$.

Hence, $\varphi^{c c}(R)=((2,4),(3,3),(1,2),(5,1),(4,5))$. 


\section{Pareto Efficiency, Individual Rationality, Strategy- Proofness, and Second-Best Incentive Compatibility}

Before introducing our main property for rules, namely second-best incentive compatibility, we introduce and discuss some well-known properties for rules. First we consider an efficiency requirement.

Pareto Efficiency: for all $R \in \mathcal{R}^{N}$ there exists no $y \in X$ such that for all $i \in N$, $y_{i} R_{i} \varphi_{i}(R)$, and for some $j \in N, y_{j} P_{j} \varphi_{j}(R)$.

Second, we formulate a voluntary participation condition: no agent receives a bundle that he considers worse than his endowment.

Individual Rationality: for all $R \in \mathcal{R}^{N}$ and all $i \in N, \varphi_{i}(R) R_{i}(i, \ldots, i)$.

Next, we discuss an incentive property: no agent ever benefits from misrepresenting his preference relation. In game theoretical terms, a rule is strategy-proof if in its associated direct revelation game form, it is a weakly dominant strategy for each agent to announce his true preference relation. Given $i \in N, R \in \mathcal{R}^{N}$, and $R_{i}^{\prime} \in \mathcal{R}$, we denote by $\left(R_{i}^{\prime}, R_{-i}\right) \in \mathcal{R}^{N}$ the new profile that is obtained from $R$ by replacing $R_{i}$ with $R_{i}^{\prime}$.

Strategy-Proofness: for all $R \in \mathcal{R}^{N}$, all $i \in N$, and all $R_{i}^{\prime} \in \mathcal{R}, \varphi_{i}(R) R_{i} \varphi_{i}\left(R_{i}^{\prime}, R_{-i}\right)$.

Ma (1994) proved that for housing markets with one object type, the core rule $\varphi^{c c}$ is the unique rule satisfying Pareto efficiency, individual rationality, and strategy-proofness. For multiple-type housing markets with more than one object type, however, Konishi et al. (2001, Proposition 4.1) showed that no Pareto efficient, individually rational, and strategy-proof rule exists. Given this impossibility, Miyagawa (2002b) demonstrated that by weakening Pareto efficiency and by strengthening strategy-proofness an alternative characterization of the core for housing markets with one object type can be obtained that does extend to multiple-type housing markets: the coordinate-wise core rule $\varphi^{c c}$ is the unique rule satisfying citizen sovereignty ${ }^{4}$, individual rationality, and strong strategyproofness $^{5}$ (see Miyagawa, 2002b, Theorem 1). Wako (2005, Theorem 1) considered a normal form game and showed that its unique coalition-proof equilibrium outcome equals the coordinate-wise core. Thus, even though the coordinate-wise core rule $\varphi^{c c}$ is not Pareto efficient, it has many appealing properties. We prove another appealing property of the coordinate-wise core rule $\varphi^{c c}$ : no other strategy-proof rule Pareto dominates $\varphi^{c c}$.

Pareto Domination of Rules: Rule $\psi$ Pareto dominates rule $\varphi$ if for all $R \in \mathcal{R}^{N}$ and all $i \in N, \psi_{i}(R) R_{i} \varphi_{i}(R)$ and for some $R^{\prime} \in \mathcal{R}^{N}$ and $j \in N, \psi_{j}\left(R^{\prime}\right) P_{j}^{\prime} \varphi_{j}\left(R^{\prime}\right)$.

Second-Best Incentive Compatibility: If rule $\varphi$ is strategy-proof and no strategyproof rule $\psi$ Pareto dominates rule $\varphi$, then $\varphi$ is second-best incentive compatible.

Theorem 1. The coordinate-wise core rule $\varphi^{c c}$ is second-best incentive compatible.

We use the following lemma in the proof of Theorem 1. Basically it states that if at some profile an allocation $y$ Pareto dominates the coordinate-wise core allocation $x$, then some agent who prefers $y$ to $x$ must receive in some marginal object type market an object at $y$ that, according to marginal preferences, is worse than the one received at $x$.

\footnotetext{
${ }^{4} \mathrm{~A}$ rule $\varphi$ satisfies citizen sovereignty if no allocation is excluded from the range of the trading rule, i.e., for all $x \in X$ there exists $R \in \mathcal{R}^{N}$ such that $\varphi(R)=x$.

${ }^{5} \mathrm{~A}$ rule $\varphi$ satisfies strong strategy-proofness if it is strategy-proof and non-bossy, i.e., for all $R \in \mathcal{R}^{N}$, $i \in N$, and $R_{i}^{\prime} \in \mathcal{R}$, there exists no $S \subseteq N$ with $i \in S$ such that for all $j \in S, \varphi_{j}\left(R_{i}^{\prime}, R_{-i}\right) R_{j} \varphi_{j}(R)$ and for some $k \in S, \varphi_{k}\left(R_{i}^{\prime}, R_{-i}\right) P_{k} \varphi_{k}(R)$.
} 
Lemma 1. Let $R \in \mathcal{R}^{N}$ and $x \equiv \varphi^{c c}(R)$. Let $y \in X$ such that for all $i \in N, y_{i} R_{i} x_{i}$, and for some $j \in N, y_{j} P_{j} x_{j}$. Then, there exists $k \in N$ such that $y_{k} P_{k} x_{k}$ and for some $\ell \in L$, $x_{k}(\ell) P_{k}(\ell) y_{k}(\ell)$.

Proof of Lemma 1. Let $R \in \mathcal{R}^{N}$ and $x \equiv \varphi^{c c}(R)$. Let $y \in X$ such that for all $i \in N$, $y_{i} R_{i} x_{i}$, and for some $j \in N, y_{j} P_{j} x_{j}$. Since preferences are strict,

$$
\text { for all } i \in N \text {, either } y_{i} P_{i} x_{i} \text { or } x_{i}=y_{i} \text {. }
$$

Suppose, by contradiction, that no $k \in N$ exists such that $y_{k} P_{k} x_{k}$ and for some $\ell \in L$, $x_{k}(\ell) P_{k}(\ell) y_{k}(\ell)$. Hence, by (1), separability of preferences, and strictness of marginal preferences,

$$
\text { for all } i \in N \text { and all } \ell \in L \text {, either } y_{i}(\ell) P_{i}(\ell) x_{i}(\ell) \text { or } x_{i}(\ell)=y_{i}(\ell) \text {. }
$$

Since there exists $j \in N$ such that $y_{j} P_{j} x_{j}$, by (2) there exists a marginal object type market, e.g.,$\tilde{\ell} \in L$, such that

$$
y_{j}(\tilde{\ell}) P_{j}(\tilde{\ell}) x_{j}(\tilde{\ell}) \text { and for all } i \in N, y_{i}(\tilde{\ell}) R_{i}(\tilde{\ell}) x_{i}(\tilde{\ell}) .
$$

Thus, by (3) there exists a coalition of agents that can reallocate their endowments of type $\tilde{\ell}$ among themselves such that according to their marginal preferences for objects of type $\tilde{\ell}$, they are all weakly better off and at least one member of the coalition is strictly better off. Formally, there exists a coalition $S \subseteq N$ such that $z(\tilde{\ell}) \equiv\left(y_{i}(\tilde{\ell})\right)_{i \in S} \in X_{S}(\tilde{\ell})$ and

$$
\text { for all } i \in S, z_{i}(\tilde{\ell}) R_{i}(\tilde{\ell}) x_{i}(\tilde{\ell}) \text { and for some } k \in S, z_{k}(\tilde{\ell}) P_{k}(\tilde{\ell}) x_{k}(\tilde{\ell}) \text {. }
$$

Since $x \equiv \varphi^{c c}(R),(4)$ yields the required contradiction to the definition of the (coordinatewise) core for the marginal object type market $\tilde{\ell}$.

Proof of Theorem 1. Assume, by contradiction, that there exists a strategy-proof rule $\psi$ that Pareto dominates $\varphi^{c c}$. Recall that $\varphi^{c c}$ is individually rational. Thus, since $\psi$ Pareto dominates $\varphi^{c c}$, it is individually rational as well.

In the following induction proof we transform agents' preferences such that agents prefer fewer and fewer object types to their respective object type endowments. For $R \in \mathcal{R}^{N}$ and $i \in N, B\left(R_{i}\right) \equiv \sum_{\ell \in L}\left|\left\{j \in N: j P_{i}(\ell) i\right\}\right|$ equals the total number of object types that agent $i$ prefers to his respective object type endowments. We denote the total number of object types that agents prefer to their respective object type endowments by $B(R) \equiv \sum_{i \in N} B\left(R_{i}\right)$.

Induction Basis (Induction Step 0): Since $\psi$ Pareto dominates $\varphi^{c c}$, for all $R \in \mathcal{R}^{N}$ and all $i \in N, \psi_{i}(R) R_{i} \varphi_{i}^{c c}(R)$ and for some $R^{0} \in \mathcal{R}^{N}$ and $j \in N, \psi_{j}\left(R^{0}\right) P_{j}^{0} \varphi_{j}^{c c}\left(R^{0}\right)$. To simplify notation let $x^{0} \equiv \varphi^{c c}\left(R^{0}\right)$ and $y^{0} \equiv \psi\left(R^{0}\right)$. By Lemma 1 , there exists $j(0) \in N$ such that $y_{j(0)}^{0} P_{j(0)}^{0} x_{j(0)}^{0}$ and for some $\ell(0) \in L, x_{j(0)}^{0}(\ell(0)) P_{j(0)}^{0}(\ell(0)) y_{j(0)}^{0}(\ell(0))$. We now change agent $j(0)$ 's preferences $R_{j(0)}^{0}$ to preferences $R_{j(0)}^{1} \in \mathcal{R}$ such that:

(i) According to $j(0)$ 's new marginal preferences $R_{j(0)}^{1}(\ell)$ for any object type $\ell \in L, y_{j(0)}^{0}(\ell)$ is the best object of type $\ell$ and if it is different from agent $j(0)$ 's endowment of type $\ell$, then (the endowment of type $\ell$ ) $j(0)$ is the second best object of type $\ell$, i.e., for all $\ell \in L$, $y_{j(0)}^{0}(\ell) R_{j(0)}^{1}(\ell) j(0)$ and for all $i \in N \backslash\left\{y_{j(0)}^{0}(\ell), j(0)\right\}, j(0) P_{j(0)}^{1}(\ell) i$. 
(ii) Any commodity bundle $z_{j(0)} \in N^{L}$ that assigns an object of type $\ell \in L$ that does not equal $y_{j(0)}^{0}(\ell)$ or the endowment of type $\ell$ is worse than the endowment, i.e., for all $z_{j(0)} \in N^{L}$ such that for some $\hat{\ell} \in L, z_{j(0)}(\hat{\ell}) \in N \backslash\left\{y_{j(0)}^{0}(\hat{\ell}), j(0)\right\},(j(0), \ldots, j(0)) P_{j(0)}^{1} z_{j(0)}$. Let $R^{1} \equiv\left(R_{j(0)}^{1}, R_{-j(0)}^{0}\right) \in \mathcal{R}^{N}$. To simplify notation let $x^{1} \equiv \varphi^{c c}\left(R^{1}\right)$ and $y^{1} \equiv \psi\left(R^{1}\right)$. Note that at $R_{j(0)}^{1}$ agent $j(0)$ 's best bundle equals $y_{j(0)}^{0}$. Thus, by strategy-proofness of $\psi, y_{j(0)}^{1}=y_{j(0)}^{0}$. By strategy-proofness of $\varphi^{c c}, x_{j(0)}^{1} \neq y_{j(0)}^{0}$. Hence, $x_{j(0)}^{1} \neq y_{j(0)}^{1}$. Then, since $\psi$ Pareto dominates $\varphi^{c c}, y_{j(0)}^{1} P_{j(0)}^{1} x_{j(0)}^{1}$ and for all $i \in N, y_{i}^{1} R_{i} x_{i}^{1}$. Thus, by Lemma 1 , there exists $j(1) \in N$ such that $y_{j(1)}^{1} P_{j(1)}^{1} x_{j(1)}^{1}$ and for some $\ell(1) \in L$, $x_{j(1)}^{1}(\ell(1)) P_{j(1)}^{1}(\ell(1)) y_{j(1)}^{1}(\ell(1))$.

Claim 1: $j(1) \in N \backslash\{j(0)\}$

Proof of Claim 1: If $j(1)=j(0)$, then $x_{j(0)}^{1}(\ell(1)) P_{j(0)}^{1}(\ell(1)) y_{j(0)}^{1}(\ell(1))$ contradicts that according to $j(0)$ 's new marginal preferences $R_{j(0)}^{1}(\ell(1)), y_{j(0)}^{1}(\ell(1))\left[=y_{1}^{0}(\ell(1))\right]$ is the best object of type $\ell(1)$. Hence, $j(1) \neq j(0)$.

Define $N(1) \equiv|N \backslash\{j(0)\}|$. Note that $N(1)<|N|$.

Induction Step $k$ : Let $k \geq 1$ and $j(k) \in N$ such that $y_{j(k)}^{k} P_{j(k)}^{k} x_{j(k)}^{k}$ and for some $\ell(k) \in L, x_{j(k)}^{k}(\ell(k)) P_{j(k)}^{k}(\ell(k)) y_{j(k)}^{k}(\ell(k))$. We now change agent $j(k)$ 's preferences $R_{j(k)}^{k}$ to preferences $R_{j(k)}^{k+1} \in \mathcal{R}$ such that:

(i) According to $j(k)$ 's new marginal preferences $R_{j(k)}^{k+1}(\ell)$ for any object type $\ell \in L, y_{j(k)}^{k}(\ell)$ is the best object of type $\ell$ and if it is different from agent $j(k)$ 's endowment of type $\ell$, then (the endowment of type $\ell$ ) $j(k)$ is the second best object of type $\ell$, i.e., for all $\ell \in L$, $y_{j(k)}^{k}(\ell) R_{j(k)}^{k+1}(\ell) j(k)$ and for all $i \in N \backslash\left\{y_{j(k)}^{k}(\ell), j(k)\right\}, j(k) P_{j(k)}^{k+1}(\ell) i$.

(ii) Any commodity bundle $z_{j(k)} \in N^{L}$ that assigns an object of type $\ell \in L$ that does not equal $y_{j(k)}^{k}(\ell)$ or the endowment of type $\ell$ is worse than the endowment, i.e., for all $z_{j(k)} \in$ $N^{L}$ such that for some $\hat{\ell} \in L, z_{j(k)}(\hat{\ell}) \in N \backslash\left\{y_{j(k)}^{k}(\hat{\ell}), j(k)\right\},(j(k), \ldots, j(k)) P_{j(k)}^{k+1} z_{j(k)}$.

Let $R^{k+1} \equiv\left(R_{j(k)}^{k+1}, R_{-j(k)}^{k}\right) \in \mathcal{R}^{N}$. To simplify notation let $x^{k+1} \equiv \varphi^{c c}\left(R^{k+1}\right)$ and $y^{k+1} \equiv \psi\left(R^{k+1}\right)$. Note that at $R_{j(k)}^{k}$ agent $j(k)$ 's best bundle equals $y_{j(k)}^{k}$. Similarly as before it follows that $y_{j(k)}^{k+1}=y_{j(k)}^{k}, x_{j(k)}^{k+1} \neq y_{j(k)}^{k}$, and $y_{j(k)}^{k+1} P_{j(k)}^{k+1} x_{j(k)}^{k+1}$. By Lemma 1, there exists $j(k+1) \in N$ such that $y_{j(k+1)}^{k+1} P_{j(k+1)}^{k+1} x_{j(k+1)}^{k+1}$ and for some $\ell(k+1) \in L$, $x_{j(k+1)}^{k+1}(\ell(k+1)) P_{j(k+1)}^{k+1}(\ell(k+1)) y_{j(k+1)}^{k+1}(\ell(k+1))$.

Claim $k+1: j(k+1) \in N \backslash\{j(0), \ldots, j(k)\}$ or $B\left(R_{j(k+1)}^{k}\right)>B\left(R_{j(k+1)}^{k+1}\right)$

Proof of Claim $k+1$ : Suppose that $j(k+1) \in\{j(0), \ldots, j(k)\}$, i.e., for some $k \geq k^{\prime} \geq 0$, $j(k+1)=j\left(k^{\prime}\right)$. Hence, $R_{j(k+1)}^{k}$ resulted from a previous transformation. Without loss of generality, at the end of Step $k^{\prime}-1, R_{j(k+1)}^{k}=R_{j(k+1)}^{k^{\prime}}$. Thus, $B\left(R_{j(k+1)}^{k}\right)=B\left(R_{j(k+1)}^{k^{\prime}}\right)=$ $\sum_{\ell \in L}\left|\left\{y_{j(k+1)}^{k^{\prime}}(\ell): y_{j(k+1)}^{k^{\prime}}(\ell) \neq j(k+1)\right\}\right|$. By $(i i)$, for all $\ell \in L, y_{j(k+1)}^{k}(\ell) \in\left\{y_{j(k+1)}^{k^{\prime}}(\ell)\right.$ : $\left.y_{j(k+1)}^{k^{\prime}}(\ell) \neq j(k+1)\right\}$. Since for some $\ell(k+1) \in L, x_{j(k+1)}^{k+1}(\ell(k+1)) P_{j(k+1)}^{k+1}(\ell(k+1))$ $y_{j(k+1)}^{k+1}(\ell(k+1)), B\left(R_{j(k+1)}^{k}\right)>B\left(R_{j(k+1)}^{k+1}\right)$.

Define $N(k+1) \equiv|N \backslash\{j(0), \ldots, j(k)\}|$.

Note that at the end of each Induction Step $k, N(k)>N(k+1)$ or $B\left(R^{k}\right)>B\left(R^{k+1}\right)$. Hence, after finitely many induction steps $\hat{k}, N(\hat{k}+1)=0$ or $B\left(R^{\hat{k}+1}\right)=0$. If $N(\hat{k}+1)=0$, then in a contradiction to Lemma 1 no further agent $j(\hat{k}+1) \in N \backslash\{1, \ldots, j(\hat{k})\}$ exists at 
the end of Step $\hat{k}$. Hence, $B\left(R^{\hat{k}+1}\right)=0$. Then, by individual rationality, for all $i \in N$, $x_{i}^{\hat{k}+1}=y_{i}^{\hat{k}+1}=(i, \ldots, i)$. However, at the end of Step $\hat{k}$, there exists $j(\hat{k}+1) \in N$ such that $x_{j(\hat{k}+1)}^{\hat{k}+1} \neq y_{j(\hat{k}+1)}^{\hat{k}+1}$; a contradiction.

\section{Remark 1. Anonymity and Neutrality}

Note that in the top trading algorithm neither the names of the objects nor the names of the agents play any particular role. This implies that in addition to all previously discussed properties, the coordinate-wise core also satisfies neutrality and anonymity.

\section{Remark 2. Uniqueness}

One can easily show that the coordinate-wise core is not the only second-best incentive compatible rule.

Consider the following slight variation of the coordinate-wise core. For simplicity assume that $\bar{\ell}=1$. Fix two agents, without loss of generality, agents 1 and 2 , with the specification that agent 2 can never receive agent 1's endowment when applying the top trading algorithm. Then, for any profile $R \in \mathcal{R}^{N}$ we calculate $\varphi^{1,2}(R)$ by applying the top trading algorithm with the extra specification that agent 2 is not allowed to point to agent 1. Formally, we define $\varphi^{1,2}$ as follows. Let $R \in \mathcal{R}^{N}$ and $R^{1,2} \equiv\left(\bar{R}_{2}, R_{-2}\right)$ where $\bar{R}_{2}$ is such that for all $j \neq 1, j \bar{P}_{2} 1$, and for all $j, k \neq 1, j \bar{R}_{2} k$ if and only if $j R_{2} k$ ( $\bar{R}_{2}$ is obtained from $R_{2}$ by making object 1 the worst object without changing preferences concerning other objects). Then, $\varphi^{1,2}(R) \equiv \varphi^{c c}\left(R^{1,2}\right)$. Loosely speaking, the second-best incentive compatibility of $\varphi^{c c}$ and the fact that for many $R \in \mathcal{R}^{N}$ such that $\varphi^{1,2}(R) \neq \varphi^{c c}(R)$, agent 2's trade restriction benefits some other agent(s), imply that $\varphi^{1,2}$ is second-best incentive compatible. Note that $\varphi^{1,2}$ can easily be (coordinate-wise) extended to $\bar{\ell}>1$. Clearly, $\varphi^{1,2}$ is individually rational, but neither Pareto efficient $\left(\varphi^{1,2}\right.$ does also not satisfy the weaker requirements of citizen sovereignty) nor anonymous.

Another class of rules that are second-best incentive compatible because they are all Pareto efficient and strategy-proof (but not individually rational!) are serial dictatorship rules: the first agent in a fixed order chooses his favorite bundle, then the second agent chooses his favorite bundle among the remaining feasible bundles, etc. In fact, also dictatorial rules where the choice of the next chooser may depend on previous choices, object type combinations previously chosen, identity of previous choosers, etc., are Pareto efficient and strategy-proof and therefore second-best incentive compatible.

It is an open problem if, apart from the coordinate-wise core rule there are other individually rational, strategy-proof, anonymous (neutral), and second-best incentive compatible rules: the so-called top-trading rule where agents are only allowed to trade their (complete) endowments is individually rational, strategy-proof, anonymous, and neutral. Conjecture: The top-trading rule is second-best incentive compatible. 


\section{References}

Abdulkadiroğlu, A., Sönmez, T. (1999) "House Allocation with Existing Tenants," Journal of Economic Theory 88, 233-260.

Chen, Y., Sönmez, T. (2004) "An Experimental Study of House Allocation Mechanisms," Economics Letters 83, 137-140.

Chen, Y., Sönmez, T. (2002) "Improving Efficiency of On-Campus Housing: An Experimental Study," American Economic Review 92, 1669-1686.

Konishi, H., Quint, T., Wako, J. (2001) "On the Shapley-Scarf Market: the Case of Multiple Indivisible Goods," Journal of Mathematical Economics 35, 1-15.

Ma, J. (1994) "Strategy-Proofness and the Strict Core in a Market with Indivisibilities," International Journal of Game Theory 23, 75-83.

Miyagawa, E. (2002a) "Strategy-Proofness and the Core in House Allocation Problems," Games and Economic Behavior 38, 347-361.

Miyagawa, E. (2002b) "Strategy-Proofness for the Reallocation of Multiple Types of Indivisible Goods," Working Paper August 2002.

Moulin, H. (1995) Cooperative Microeconomics: A Game Theoretic Introduction, Princeton, NJ: Princeton University Press.

Roth, A.E. (1982) "Incentive Compatibility in a Market with Indivisible Goods," Economics Letters $\mathbf{9}, 127-132$.

Roth, A.E., Postlewaite, A. (1977) "Weak versus Strong Domination in a Market with Indivisible Goods," Journal of Mathematical Economics 4, 131-137.

Shapley, L., Scarf, H. (1974) "On Cores and Indivisibility," Journal of Mathematical Economics 1, 23-37.

Sönmez, T. (1999) "Strategy-Proofness and Essentially Single-Valued Cores," Econometrica 67, 677-689.

Sönmez, T., Ünver, U. (2005) "House Allocation with Existing Tenants: An Equivalence," Games and Economic Behavior 52, 153-185.

Wako, J. (2005) "Coalition-Proof Nash Allocation in a Barter Game with Multiple Indivisible Goods," Mathematical Social Sciences 49, 179-199. 